\title{
AdaptFlow: Protocol-based Medical Treatment Using Adaptive Workflows
}

\author{
U. Greiner', R. Mueller', E. Rahm', J. Ramsch², B. Heller², M. Loeffler² \\ 'Department of Computer Science, University of Leipzig, Germany \\ ${ }^{2}$ Institute for Medical Informatics, Statistics and Epidemiology, University of Leipzig, Germany
}

\begin{abstract}
Summary
Objectives: In many medical domains investigatorinitiated clinical trials are used to introduce new treatments and hence act as implementations of guidelinebased therapies. Trial protocols contain detailed instructions to conduct the therapy and additionally specify reactions to exceptional situations (for instance an infection or a toxicity). To increase quality in health care and raise the number of patients treated according to trial protocols, a consultation system is needed that supports the handling of the complex trial therapy processes efficiently. Our objective was to design and evaluate a consultation system that should 1) observe the status of the therapies currently being applied, 2) offer automatic recognition of exceptional situations and appropriate decision support and 3) provide an automatic adaptation of affected therapy processes to handle exceptional situations.

Methods: We applied a hybrid approach that combines process support for the timely and efficient execution of the therapy processes as offered by workflow management systems with a knowledge and rule base and a mechanism for dynamic workflow adaptation to change running therapy processes if induced by changed patient condition

Results and Conclusions: This approach has been implemented in the AdaptFlow prototype. We performed several evaluation studies on the practicability of the approach and the usefulness of the system. These studies show that the AdaptFlow prototype offers adequate support for the execution of real-world investigator-initiated trial protocols and is able to handle a large number of exceptions.
\end{abstract}

\section{Keywords}

Clinical trials, clinical protocols, guidelines, eventoriented exception handling, adaptive workflow management

Methods Inf Med 2005; 44: 80-8

\section{Introduction}

Clinical trials are the accepted method to evaluate new therapies in medicine $[1,2]$. They allow the compatibility and the efficiency of new therapies to be examined and offer a way to compare different treatments for the same disease pattern. Investigatorinitiated multicenter trial treatment protocols are particularly widespread in hematooncology and often encompass several thousand patients. Hence, although they are designed primarily to provide answers to scientific questions they often also act as guidelines for physicians in many hospitals to specify the treatment(s). In Germany about $20-50 \%$ (disease-dependent) of all newly diagnosed patients with leukemias or lymphomas will be included in a trial. The protocols usually contain detailed instructions on how to perform diagnosis, therapy, toxicity management, and follow-up (see section 3). In our institution we are responsible for the biometry and coordination of many such trials.

One goal for the use of investigator-initiated treatment protocols is to increase the quality in health care. In order to raise the number of patients treated according to high-quality treatment protocols and taking part in clinical trials, a consultation system is needed that supports the handling of the entire complex trial therapy processes efficiently. To support clinicians in selecting the adequate trials and treatments in oncology we have designed a web-based software tool called OncoWorkstation. It provides assistance in the choice, comparison, and planning of oncological guideline-based trial protocols [3]. However, the execution support is limited to simple calculations of patient-related therapy plans containing the drug applications. Despite the detailed ther- apy plans, a significant degree of flexibility in treatments arises through exceptional situations (e.g., an infection or a toxicity) which may frequently occur during therapy execution. For instance, we checked anonymous patient data ${ }^{a}$ for a high dosage lymphoma protocol (MEGA-CHOEP [4]) and found that for nearly each patient at least one treatment cycle had to be postponed mostly due to acute toxicity. Furthermore, more than $11 \%$ of the patients dropped out of the trial during the treatment and for further $11 \%$ of the patients the protocol treatment has been abandoned (see Table 1).

Treatment protocols additionally contain information about the proper reactions to such exceptional situations. However, a specialized physician has to handle many different protocols for different patients at the same time. Therefore, a convenient consultation system should observe the status of the therapies currently being applied, offer automatic recognition of exceptional situations and appropriate decision support for handling such situations. Furthermore, the system should be able to automatically adapt affected therapy processes to adequately handle the flexibility of treatment processes.

For some of these problems, e.g., the handling of data and knowledge or the organization of clinical activities, there are already several systems in use in the medical domain [5-10]. But to meet all these requirements, a hybrid approach is necessary that combines a workflow management system with a knowledge and rule base. The workflow system supports the coordination of activities and the integration of different users,

\footnotetext{
The data has been kindly provided by the Non-Hodgkin-Lymphoma study secretariat at the Institute for Medical Informatics, Statistics and Epidemiology, University of Leipzig (http;//www. lymphome.de/Gruppen/DSHNHL).
} 
data and applications $[11,12]$ to ensure the timely and efficient execution of therapy processes. It is used to model the therapy processes and coordinate their execution by notifying each user (e.g., a physician or a nurse) in time about the next therapy steps he has to execute for a particular patient treated according to a particular protocol. The workflow system also provides the user with the data needed for each therapy step. Furthermore, the workflow system has to offer dynamic adaptation of therapy workflows during execution to handle the flexibility of therapy processes $[13,14]$, i.e. to change running therapy processes if induced by changed patient condition. Thus the therapy processes may be adapted to a changed situation and always represent the appropriate therapy for a particular patient. The rule base handles the medical knowledge represented in the protocols and is used to detect exceptional situations.

\section{Objectives}

The hybrid approach has been implemented in the AdaptFlow prototype that is being developed at the University of Leipzig. The main objectives of the AdaptFlow prototype are:

- observation of the status of the therapies currently being applied,

- a novel ECA (Event/Condition/Action) model [15] to describe the exceptional situations formally,

- automatic recognition of exceptional situations that may require changes of treatment,

- appropriate decision support to determine the suitable therapy changes in consideration of the trial protocol and the appropriate changes of the treatment workflow, and

- automatic adaptation of affected therapy processes so that they can be executed further.

Furthermore, AdaptFlow is to offer the user the possibility to modify the therapy manually in case of unexpected exceptions or if the automatically-derived changes are undesired. To cover the complexity of different protocols, three representative trial proto-
Table 1 Sample figures for the frequency of selected exceptions in the adult highly malignant non-Hodgkin lymphoma high dosage therapy MEGA-CHOEP [4]

\begin{tabular}{|l|l|}
\hline $\begin{array}{l}\text { Exception } \\
\text { postponement of therapy } \\
\text { cycle }\end{array}$ & Frequency \\
\hline $\begin{array}{l}\text { postponement of therapy cy- } \\
\text { cle due to high toxicity de- } 273 \text { patients } \\
\text { gree }\end{array}$ & $\begin{array}{l}19.8 \% \text { of } 5648 \text { toxicity } \\
\text { measurements performed } \\
\text { for } 273 \text { patients }\end{array}$ \\
\hline $\begin{array}{l}\text { premature end of therapy } \\
\text { premature end of protocol } \\
\text { conform treatment }\end{array}$ & $11 \%$ of 273 patients \\
\hline
\end{tabular}

cols from different oncological fields have been chosen: high dosage therapy used in aggressive non-Hodgkin lymphoma (MEGA-CHOEP [4]), adult colon cancer therapy (InTact [16]) and pediatric acute myeloid leukemia therapy (AML-BFM [17]). The focus is on protocols from oncology as they offer long running processes suitable for workflow management, a high degree of relevance and complexity, and a significant number of exceptional situations that are described in the therapy protocols.

The paper is organized as follows. Section 2 discusses related work. Section 3 describes a medical scenario for a therapy protocol before providing an exception classification and introducing the rule model in section 4. Section 5 gives an overview of the AdaptFlow prototype and section 6 presents the evaluation. A discussion is subject of section 7 before we close with a summary and a sketch of future work (section 8).

\section{Related Work}

The application scenario for the AdaptFlow prototype is the enhancement of consultation systems with workflow support for the execution of complete therapy processes described in investigator-initiated trial protocols. Thus, AdaptFlow is not part of a clinical information system as described in [8-10]. However, it is suitable to complement clinical information systems, especially to provide support for therapy processes. It is also not a laboratory system or a calculator for therapy plans but uses the functionality offered by these systems to support the execution of the entire therapy process. For instance, data provided by chemotherapy calculation programs may be presented to the user together with the activities in a process instance for a chemotherapy cycle.

AdaptFlow also differs from expert systems for therapy planning and monitoring such as ONCOCIN [18] as it does not use planning techniques to iteratively calculate the therapy plans but uses predefined therapy processes represented as workflows. To handle the inherent flexibility the workflows are dynamically adapted if failure events occur. The necessary modifications are derived from the rules contained in the investigator initiated trial protocols again without the use of planning or refinement techniques from artificial intelligence.

Several vendors and researchers have addressed failure and exception handling in workflow management systems [19-25] or in computerized implementation of clinical guidelines [26-28]. However, only a few commercial workflow systems such as [19-21] provide any support for workflow adaptation. For example, [21] is able to derive on the basis of ECA rules that an additional activity has to be executed. However, the user has to select an appropriate insertion point in the workflow manually. Furthermore, most commercial systems do not offer decision support for the choice of the appropriate type of exception handling.

Some research prototypes offer manual workflow adaptations or automatic adaptation of the activity currently being executed [22-24]. The first approach requires an expert who has to decide which events (e.g. laboratory values) constitute logical failures and which adaptations have to be performed. However, considering the huge amount of data a physician is faced with, this approach can be time-consuming and error-prone as exceptional situations may be detected too late or overlooked completely.

The second approach is also only of limited usefulness as workflow parts not yet executed cannot be adapted. In oncology, this is of particular importance as the patientspecific drugs for a chemotherapy have to be ordered one or two days before the plan- 


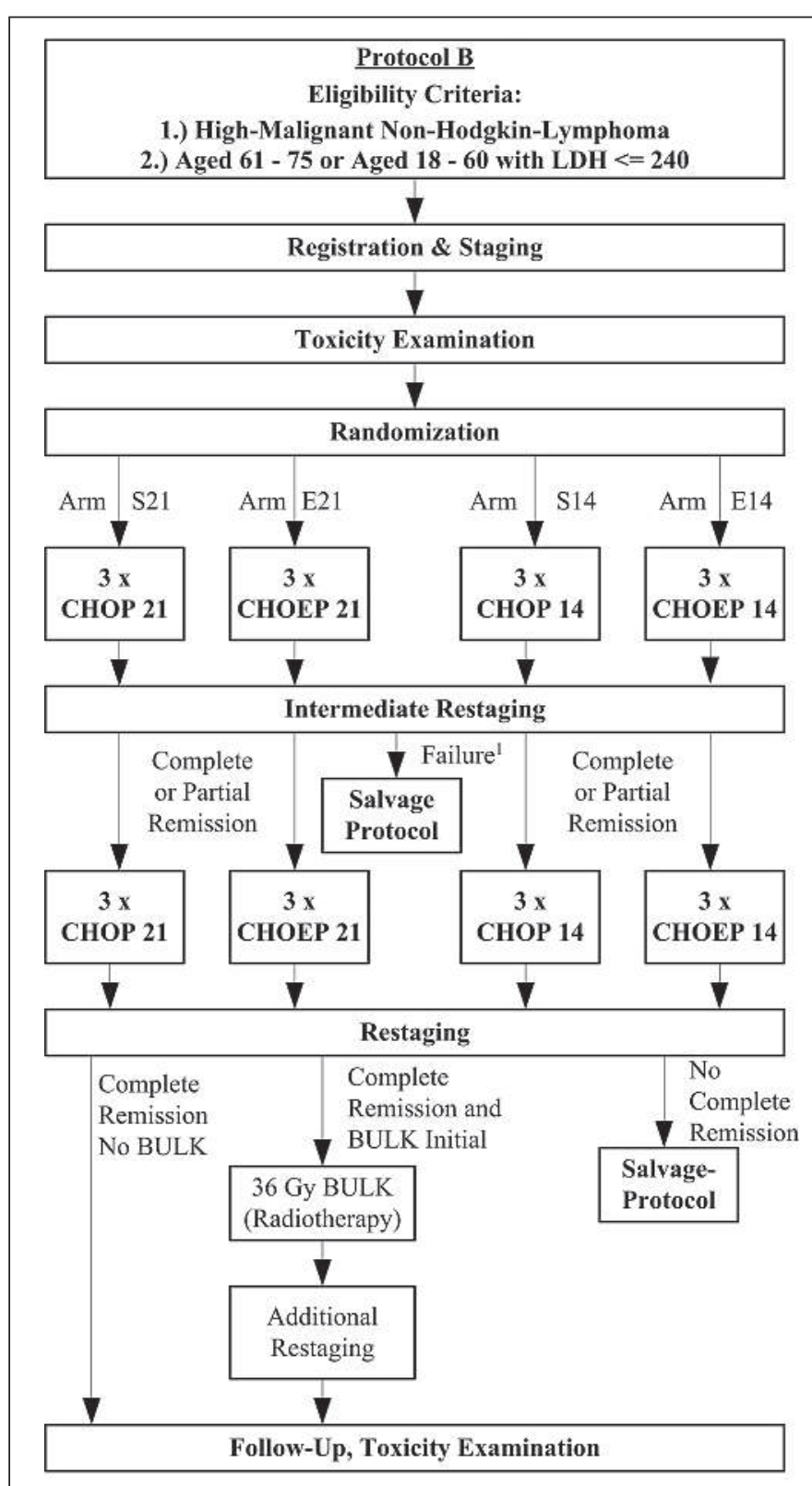

${ }^{1}$ Failure $=$ Progression, No Change, Minor Response of Tumor
Fig. 1

Flow sheet for NHL-B protocol [42]. (The rectangles represent the therapy steps, the labels encode the type of examination to be performed or the chemotherapy to be applied (e.g. CHOP 21). Each step is documented on appropriate case report forms.) ned administration. Thus, in order to allow for the timely administration of drugs or to prevent an infusion from having to be discarded, the corresponding workflow adaptation should be performed as soon as possible.

Medical planning systems (e.g., [27, 29, 30 ] in oncology) offer an adequate representation of medical knowledge, but typically do not support the temporal dimension of failures sufficiently and offer only limited support of operational aspects. For instance, workflow modeling and execution is not supported efficiently. So, existing processes cannot easily be changed if the underlying protocols are changed. Furthermore, the systems do not offer advanced integration of data and users.

\section{Scenario}

A common way to describe a chemotherapeutical therapy is to display the normal therapy process with its prerequisites (i.e. the conditions under which a patient is eligible for the therapy and fulfills the requirements of the trial) and the circumstances under which an alternative behavior should be employed or under which a trial-compliant treatment is no longer possible. An example is shown in Figure 1. Chemotherapies typically consist of several main therapy processes (called cycles) in different therapy branches (e.g., in Fig. 1 CHOP21 denotes a cycle). The cycles are separated by staging and restaging examinations, documentation steps and additional therapeutic steps such as radio or supportive therapies. Some of the therapy components (e.g. the cycles) can be decomposed into smaller parts such as the administrations of drugs or the examination of diagnostic parameters. These parts are encoded as activities within the workflow definition. Figure 2 shows a fragment of a therapy cycle consisting of five drug administration activities executed in parallel (application details have been omitted for a clearer presentation).

Oncological therapies are complex and their process may differ from patient to patient, even if they have the same disease. However, the necessity of a treatment modification for a particular patient often does not reveal itself until the treatment is already in progress. The changes are triggered through events (exceptions) such as allergies, low blood counts, or unexpected disease progression requiring adaptations such as dosage modifications, drug replacements or individual supportive therapy steps. Normally the treating physician chooses the most appropriate out of several medically and economically feasible and ethically justifiable alternatives. For treatments in clinical trials, the adaptations needed for important and frequently occurring exceptions are described in the therapy protocol.

To illustrate how a therapy workflow is adapted when an exceptional situation arises, consider the following scenario. During the execution of some preliminary activities a neurological toxicity (Event 1 in Fig. 2) and a hematological toxicity (Event 2 in Fig. 2) are detected. Hence, the chemotherapy cannot be applied according to the workflow definition and the workflow has to be adapted in the ranges specified in the 


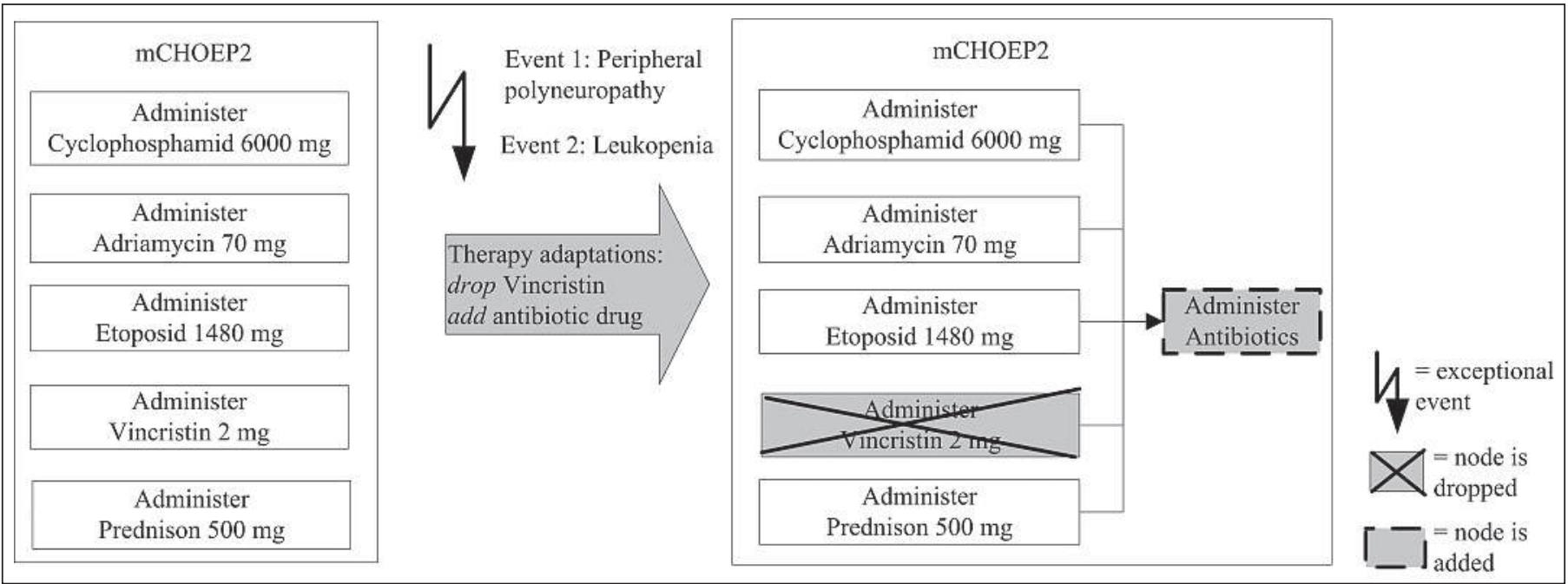

Fig. 2 Logical failure handling in chemotherapy scenario

protocol. Since e.g. Vincristin is known to cause neurological toxicities, the activity Administer Vincristin has to be dynamically removed while the execution of the other activities can be continued without change. Furthermore, antibiotic drugs have to be administered after the chemotherapy to compensate for the hematological toxicity and to protect the patient against infections. Thus, a new activity Administer Antibiotics has to be added to the therapy. The right fragment in Figure 2 shows the chemotherapy cycle after the adaptations have been performed.

\section{Exceptions and Rules}

We have developed a three-step concept for the automatic detection of whether a treatment adaptation is necessary, the determination of the corresponding treatment modification, and the adaptation of the treatment workflow.

To detect a situation that may require a treatment adaptation, it is necessary to establish rules that represent the conditions described in the trial protocols (see section 3 ). In order to gain a comprehensive overview of possible exceptions, we selected treatment protocols from different areas, as mentioned in section 1, and in analyzing these protocols we found exceptional events that may require treatment adaptations.
Three main event types were identified: medical events (e.g., laboratory or pathological findings, diagnoses or toxicological ratings), organizational events (e.g., resource conflicts of medical devices, changes of patient status due to hospitalization), and social events (e.g., loss of patient compliance for the treatment). Each type of event requires different data sources to be observed. Some medical events can be detected through oversight of the laboratory or other clinical databases, while for instance protocol-dependent toxicological ratings require an additional system. Most of the events described may only lead to exceptions in certain parts of the treatment. For instance, a low blood leukocyte count (in an acceptable range) may be tolerable after a
Table 2

Treatment modifications and the corresponding control actions describing the workflow adaptations. W denotes a workflow, A and $\mathrm{B}$ activities, $\mathrm{P}$ a patient, $\mathrm{t}$ a time interval, $\mathrm{p} \mathrm{a}$ parameter and $f a$ function.

\begin{tabular}{|l|l|l|}
\hline \multicolumn{1}{|l|}{ Treatment modification } & \multicolumn{2}{|l}{ Example } \\
\hline start therapy & $\begin{array}{l}\text { supportive therapy, } \\
\text { triggered by new infection }\end{array}$ & \\
\hline stop therapy & $\begin{array}{l}\text { stop until patient status has } \\
\text { normalized, triggered e.g.r } \\
\text { by a heart attack }\end{array}$ & suspend(W,t,P) \\
\hline abort therapy & $\begin{array}{l}\text { premature end of therapy, } \\
\text { triggered by progress of } \\
\text { disease }\end{array}$ & abort(W,P) \\
\hline postpone therapy part & $\begin{array}{l}\text { delay of drug application, } \\
\text { triggered by low leukocyte } \\
\text { count }\end{array}$ & postpone(A,t,P) \\
\hline change therapy or therapy step & $\begin{array}{l}\text { hospitalization of patient, } \\
\text { triggered by danger of } \\
\text { critical adverse reaction }\end{array}$ & change-value(A,p,f,P) \\
\hline substitute therapy step & $\begin{array}{l}\text { substitution of administered } \\
\text { drug, triggered by allergy to } \\
\text { drug }\end{array}$ & replace(A,B,P) \\
\hline add therapy step & $\begin{array}{l}\text { additional drug adminis- } \\
\text { tration, triggered by infection }\end{array}$ & add(A,P) \\
\hline delete therapy step & $\begin{array}{l}\text { cancellation of a supportive } \\
\text { therapy, triggered by discon- } \\
\text { tinuation of additional infec- } \\
\text { tion }\end{array}$ & drop(A,P) \\
\hline
\end{tabular}


cytostatic application phase, but not during other treatment phases. This illustrates the need to consider intervals of temporal validity for exception handling.

To detect exceptional events we use extended Event-Condition-Action (ECA) rules [15] that represent the conditions described in the treatment protocols (details in [31]; [32] provides a similar approach for event detection). The rules are of the following format:

\section{WHEN exceptional event \\ WITH condition \\ THEN treatment adaptation \\ VALID-TIME time period}

The event-condition part (WHEN/WITH) specifies the event and the conditions under which treatment adaptations are required. The action part (THEN) describes the necessary treatment modifications. The possible modifications are listed in Table 2. The optional valid time part (VALID-TIME or VT) specifies a time period during which the modification should be applied. It is denoted either by a fixed time interval or date, or by a conditional time interval whose end is specified by a condition that has to be satisfied (e.g., until the blood value has improved). This extension of ECA rules with temporal information is especially needed to meet medical requirements formulated in the protocols.

The following ECA rule specifies that for a patient with a critical blood value all applications of drug Vincristin should be dropped during the next seven days and a new blood examination has to be scheduled in three days (syntax simplified for better readability):

WHEN new finding for patient $P_{1}$

WITH leukocyte_serum count $<1000$ \#/ $\mathrm{mm}^{3}$

THEN drop applications of drug Vincristin for $P_{1}$ AND add new examination of leukocyte_serum count within three days

VALID-TIME during the next seven days

The modifications of medical treatments specified in the THEN-part of the rules correspond to workflow adaptations such as stopping or aborting a treatment workflow or delaying, adding, or deleting a single workflow activity. The AdaptFlow monitor- ing module (described in section 5.1 below) translates the medical treatment modifications into so-called control actions. Control actions describe the workflow adaptations on the basis of activities, i.e. single workflow tasks, or whole workflows. Each control action consists of the description of the workflow adaptation and the valid time VT as specified in the ECA rule. All control actions supported by AdaptFlow are listed in Table 2. For instance, the first modification in the THEN-part of the ECA rule shown above is translated into the control action drop (application_of_drug_Vincristin, $P_{1}$ ) @ [for next 7 days] (the '@) separates the adaptation description from the valid time part).

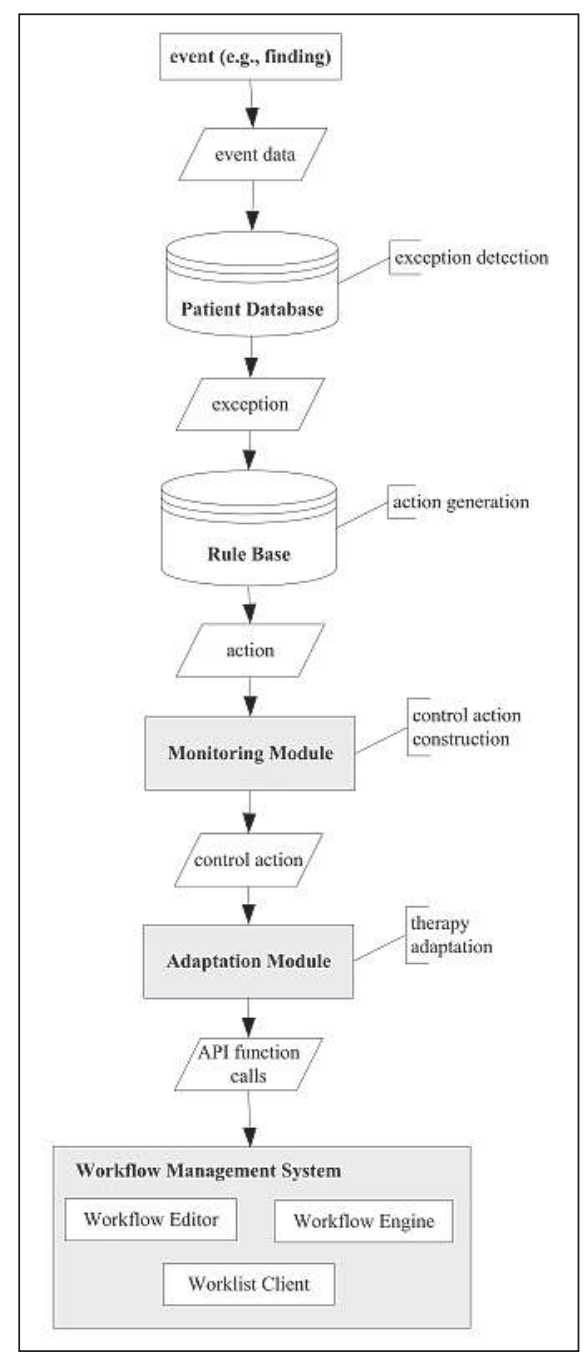

Fig. 3 AdaptFlow architecture with control flow for handling an exception

\section{The AdaptFlow Prototype}

In this section we present the AdaptFlow prototype. First the architecture is introduced before workflow adaptation is described in more detail.

\subsection{Architecture}

AdaptFlow consists of a workflow management system, a monitoring module, an adaptation module, a patient database and a rule base. Figure 3 shows these components and their interaction from the detection of an event to the workflow adaptations.

The main component of AdaptFlow is the workflow management system. Workflow management systems support the execution of clearly structured, frequently repeated processes. They offer a strict separation of application program code from the overall process logic and the integration of automated and manual activities and of data from different sources. Thus, they offer a good infrastructure for the implementation of the diagnostic and therapeutic processes of therapy protocols [13] and are used in the AdaptFlow prototype for the definition and execution of therapy workflows. As it is not suitable to develop a workflow system from scratch (due to personnel and time limitations) the $\mathrm{ADEPT}_{\text {flex }}$ workflow management system [22] is used. The workflow model of $\mathrm{ADEPT}_{\text {flex }}$ supports the specification of execution durations for activities and temporal distances between activities. Furthermore, $\mathrm{ADEPT}_{\text {flex }}$ provides operators for dropping and adding nodes in workflow instances which are called using a Java API (Application Programming Interface). This offers the possibility to perform workflow adaptations automatically, which is not supported by commercial workflow management systems or by the $\mathrm{ADEPT}_{\text {flex }}$ workflow system itself (only manual adaptations are supported).

The workflow system consists of a workflow editor for workflow modeling, a workflow engine for workflow execution and several work list clients as interfaces through which the tasks are presented to users, such 
as physicians and nurses. During workflow modeling the therapy processes described in the protocol are translated into a workflow definition, i.e. a technical representation of the therapy. If a particular therapy is to be executed for a patient, the corresponding workflow definition is instantiated by generating a workflow instance ${ }^{b}$. The workflow instance is executed by the workflow engine and the tasks are presented in the work list clients. Figure 4 shows a screenshot of an AdaptFlow work list client during the execution of a chemotherapy workflow. The upper part contains a graphical representation of the whole process. The lower part shows the activities to be executed by the user with some additional information, such as the ID of the patient for whom the activity has to be performed (first column), the start time point for the activity and the time left if it may not be started immediately (sixth and seventh column).

The patient database contains patients' master data and laboratory, clinical, and pathology findings. In our implementation it also contains database triggers to detect whether newly-entered values may constitute a logical failure event. The patient database could also be part of an existing clinical information system or from a consultation system such as OncoWorkstation.

If a critical value is detected in the patient database, i.e. a value outside the permitted range specified in the protocol, then the rule base containing medical knowledge and therapy-specific knowledge derives whether in fact the event constitutes a logical failure and determines the appropriate therapy modifications. The monitoring module translates the therapy modifications into control actions (see section 4). The control actions are transferred to the adaptation module which adapts the affected workflow instances accordingly (see 5.2).

AdaptFlow uses XML (eXtensible Markup Language) documents for the communication between its different components. XML is a widely-used data interchange format for which various communi-

b In the following, workflow will be used as a synonym for workflow instance if the context is clear.

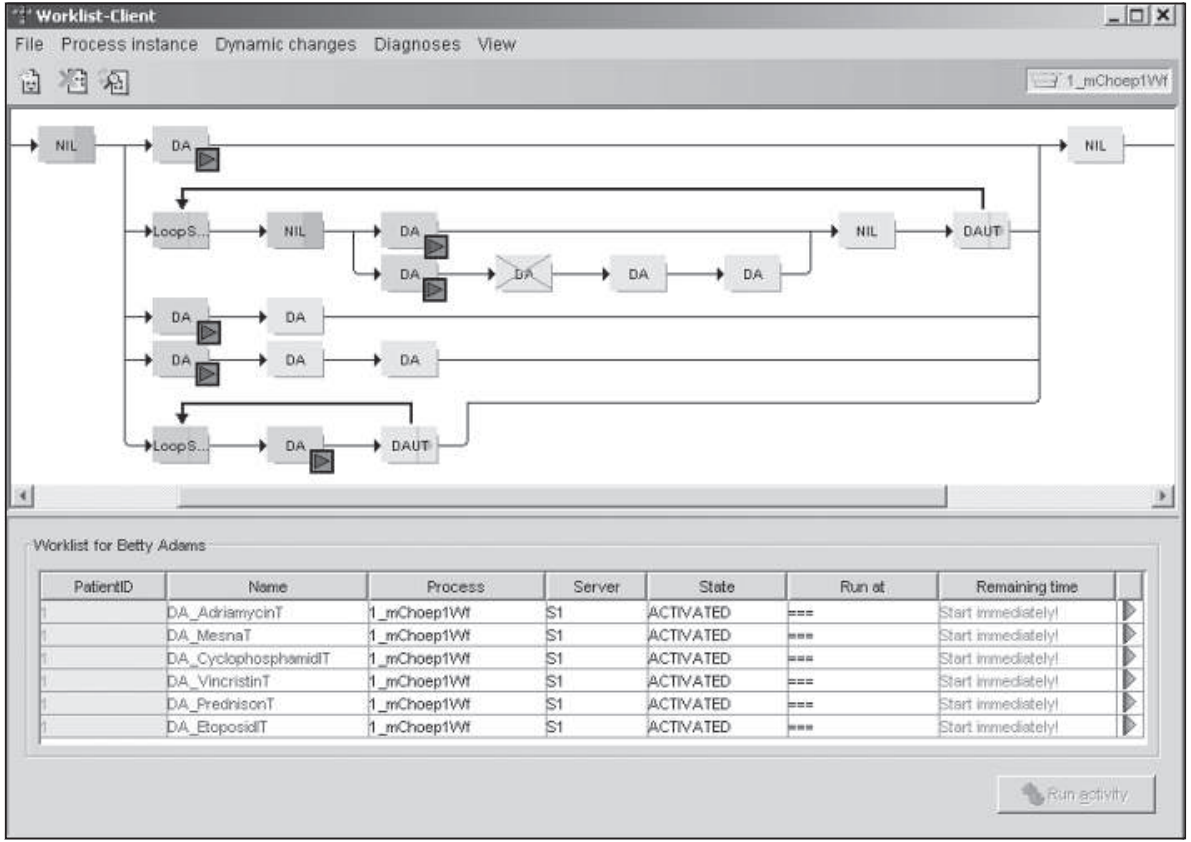

Fig. 4 Screenshot of work list client. Activities with 'DA' represent drug applications; all other activities are used to model the control flow. Activities with an attached play-button have to be executed next; all activities to the left of these have already been executed; all activities to the right of these are future activities.

cation infrastructures exist (e.g., XML-RPC which was used for the prototype).

In the current version of AdaptFlow, the rule base was implemented using advanced database functionality such as database triggers and stored procedures due to time limitations.

\subsection{Workflow Adaptation}

The workflow adaptation process follows either a predictive or a reactive adaptation strategy. If possible, workflows should be adapted predictively, i.e. as soon as an exception is detected, in order to inform workflow users in time about necessary changes, and in particular to prepare new activities (e.g., drug administrations) or cancel the preparation of deleted activities. Predictive adaptation is possible if the valid time VT of a control action is specified by a fixed (absolute) date or interval. In this case, AdaptFlow can estimate the workflow part $\mathrm{P}_{\mathrm{VT}}$ that will presumably be executed during VT and adapt it in advance using the $\mathrm{ADEPT}_{\text {flex }}$ API functions (for instance, in the screenshot in Fig. 4 the crossed-out node has been dropped).
Reactive adaptation is used if the valid time VT of a control action is denoted by a conditional time interval (e.g., until the blood value has improved), or if the workflow contains conditional parts such as conditional splits or loops. In this case it is generally not known in advance for how long the condition will hold, and as a consequence the affected activities cannot be predicted. Therefore, the affected workflow instances (i.e., all workflow instances that are currently being executed for the patient denoted in the control action) are monitored as long as the condition holds (e.g., as long as the blood leukocyte count of the patient is less than $1.000 \# / \mathrm{mm}^{3}$ ). Before an activity is scheduled to be executed, the system checks whether it is affected of the control action. If this is the case then the activity is adapted before it is executed.

All adaptations (reactive as well as predictive) must be confirmed by an authorized medical user before the modified workflow instance is resumed. Thus, the user can reject adaptations if they are not appropriate for a particular patient and perform manual exception handling instead.

The estimation of the workflow part $\mathrm{P}_{\mathrm{VT}}$ during predictive adaptation is based on 


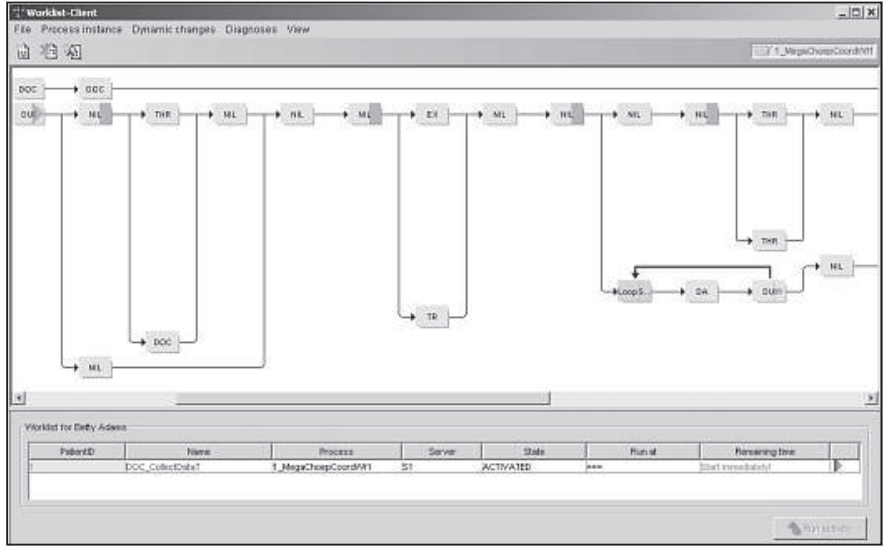

Fig. 5 Workflow instance that models the coordination workflow for the MEGA-CHOEP protocol. The screenshot shows a fragment starting with the optional pre-phase therapy (left THR-activity) and reaching up to the application of the first chemotherapy cycle which is executed in parallel to a prophylaxis therapy (the two right THR-activities). Activities with 'DA' represent drug applications; activities with 'THR' represent therapy cycles which are modeled in separate workflows; activities with ' $E X$ ' and ' $T R^{\prime}$ represent examinations and treatments; all other activities are used to model the control flow.

temporal information that was specified during workflow definition, such as average activity execution durations (e.g., the duration of a drug infusion) or temporal distances between two activities (e.g., to specify a waiting time between two drug applications or examinations). To estimate $\mathrm{P}_{\mathrm{VT}}$ of an affected workflow instance the execution durations of all paths through the remaining workflow are estimated, starting with the activities that will be executed next (for instance the activities with an attached playbutton in Fig. 4). This is done by estimating and adding together the durations of the workflow constructs (i.e., sequences, split/ join-blocks or loops) a path consists of. Estimation of a path stops if the valid time interval is "consumed" by that path or if there are irresolvable conditions at OR-SPLIT nodes or loops. $\mathrm{P}_{\mathrm{VT}}$ then consists of all nodes and edges of the estimated paths which are assumed to be executed during VT. A more detailed description of workflow estimation can be found in [31].

\section{Evaluation}

For an evaluation of the AdaptFlow prototype we have chosen a three-step approach. First, we modeled the three protocols named in section 1 in AdaptFlow to test the functionality of the system. In a second step we analyzed a set of further protocols to confirm that the hybrid approach of AdaptFlow offers adequate user support for the application of different protocols. In a third step we analyzed real patient data records to get some evidence for the practical usefulness of a system such as AdaptFlow.

\subsection{Representation of Protocols}

For the representation of the three test protocols we decided to model the different therapy cycles as separate workflows for each protocol. These are started from a coordination workflow that contains the whole therapy process. Figure 5 shows a fragment of the coordination workflow for the MEGA-CHOEP protocol, Figure 4 a fragment of a workflow for the application of the first chemotherapy cycle. The exceptions contained in the protocols were modeled using triggers on the patient database (see 5.1). Those signal the occurrence of events that may constitute an exception. Furthermore, we defined rules representing the therapy-specific knowledge about the appropriate therapy modifications for each exception.

During the protocol representation we encountered some difficulties:

- An appropriate representation of timedependent conditions is particularly important for medical therapies (e.g., to model the interval between two therapy steps). Thereby complex directives occur such as "apply drug A three times but only if all applications can be performed in this week". As the temporal model of the workflow system of AdaptFlow does not directly support such specifications we had to use workarounds for those cases which made the workflow representation more complex.

- The validity of exceptions may depend on the execution state of a workflow instance. As this often may not be derived directly from the workflow engine again the therapy process representation has to be extended, e.g., with dummy activities that just indicate a particular position in a workflow instance.

\subsection{Analysis of Further Protocols}

With the experiences made during the representation of the sample protocols we analyzed further protocols to check whether the AdaptFlow prototype is restricted to the three pre-selected protocols. We concentrated on treatment protocols from the field of oncology as they offer long running, sufficiently structured and complex processes suitable for the representation in workflow systems. Moreover, exceptions are described in the protocols to justify the use of the AdaptFlow system.

We considered the following additional protocols: three protocols for the treatment of non-Hodgkin lymphoma [33-35] and two protocols for the treatment of Hodgkin lymphoma [36, 37]. All these protocols contain chemotherapy cycles preceded, separated or followed by examinations and documentation steps. The steps of the different protocols differ in the following points:

- the type of the examinations respectively the parameters that are raised in an examination,

- the type and dosage of the applied drugs,

- the conditions that have to be fulfilled to continue the therapy,

- additional treatments, e.g. radiotherapy, in some protocols depending on the type of disease. 
These differences do not add complexity to the representation of the protocols, and AdaptFlow is able to handle them well. Also, no more complex time constructs have been found that could limit the presentability of a protocol.

The same holds for the exceptions described in the protocols. They differ in the parameters that have to be evaluated for exception detection (the parameters are provided by the examination steps of the therapy) and the thresholds that decide which modification has to be applied. As the rules used in AdaptFlow are therapy-specific, they have to be adjusted for different treatment protocols anyway. Hence, the new protocols require no additional complexity of the rule representation. A further difference between the protocols can be found w.r.t. the therapy modifications. But none of the protocols contains a therapy modification that is not supported by AdaptFlow. Thus, all additional protocols checked can be represented in AdaptFlow without any difficulties.

\subsection{Analysis of Patient Data Records}

An analysis of real patient data records at the municipal hospital "St. Georg" in Leipzig revealed the usefulness of a system such as AdaptFlow in practical clinical oncology. We looked at patients with the diagnosis lymphoma treated according to different protocols (e.g. RICOVER [38], HD10, HD11, HD12 [37]). The patients have been selected at random.

Exceptions leading to therapy modifications occurred for over $70 \%$ of the considered patients during therapy execution. Several documented exceptions were based on medical data such as toxicities, infections, diagnoses, or general ratings. Others were related to organizational or social factors. We verified that AdaptFlow is able to detect all types of exceptions that have been documented in the sample patient records.

On average, during a treatment of a single patient 3.4 exceptions leading to therapy modifications occurred. $94 \%$ of these necessary therapy modifications have been described in the therapy protocols and would therefore also have been derived by AdaptFlow.

\section{Discussion}

We conclude from the evaluation studies presented in section 6 that a significant number of therapy protocols from oncology and of exceptions contained in the protocols can be modeled, detected and handled in AdaptFlow. Thus, the combined approach of adaptive workflow management and decision support offers adequate support for the application of guideline-based treatment protocols although there are some exceptions that may not be handled in AdaptFlow. For instance, the detection of exceptions that have a broad scope of discretion may not be automated in AdaptFlow. The same holds for exceptions that are based on very complex situations that need massive user interaction for evaluation. However, these types of exceptions only make up a small fraction of all exceptions that are described in the treatment protocol. Thus, it does not reduce the advantages of the AdaptFlow system significantly.

Furthermore, due to modifications performed by earlier workflow adaptations an automatic derivation of the modifications for the next exceptions may become impossible as the structure of the workflow instance has changed significantly. So, it does not correspond anymore to the structure assumed by the rules. Nevertheless, the exceptions can still be detected automatically and the user can be informed. Thus, one main part of the AdaptFlow functionality is still available and the system is valuable for the further execution of this particular workflow instance.

Limitations for the workflow representation mainly occurred w.r.t. the limitation of existing workflow systems to model complex time constructs which are typical for therapy protocols. Thus, an enhanced time model would facilitate the workflow representation of the therapy processes.

At the moment, the necessary data for exception detection, e.g., laboratory values or diagnoses, is entered manually in Adapt-
Flow. As we need several parameters that are not directly available from existing data sources such as clinical information or laboratory systems but have to be derived manually anyway (such as toxicity degrees), this additional effort for the user is acceptable. Furthermore, integrating hospital information systems with external applications is associated with several difficulties and high costs as e.g. described in [39-41]. This integration is not addressed by the AdaptFlow prototype.

\section{Conclusions and Further Work}

The AdaptFlow prototype described in this paper offers efficient support for the handling of complex trial therapy processes by combining a workflow management system with mechanisms for rule-based dynamic workflow adaptation. AdaptFlow observes the status of the therapies currently being applied, offers automatic detection of exceptional situations and appropriate decision support as well as automatic adaptation of affected therapy processes. Thus, AdaptFlow represents an enhancement of consultation systems to facilitate treatment application for the medical staff and to increase treatment quality by enhancing the protocol conformance of the applied treatments. The AdaptFlow prototype is therefore part of a broader strategy which currently focuses on the introduction of consultation systems to support standard treatment processes. It has been fully implemented and a first evaluation has been performed. A practical use is envisioned as a second step of the overall strategy after consultation systems (such as OncoWorkstation [3]) have been widely adopted.

Future work will investigate certain aspects of workflow modeling for treatment protocols in more detail, especially modeling of temporal information such as activity execution durations. Furthermore, the optimal degree of abstraction for modeling treatment protocols has to be explored. In this context, treatment protocols from other medical domains will also be taken into account. 


\section{Acknowledgments}

This work has been supported by the German Research Association under grant Ra 497-12 which was jointly granted to Erhard Rahm and Markus Loeffler. $\mathrm{ADEPT}_{\text {flex }}$ has kindly been provided by the Dept. of Computer Science, University of Ulm. We would like to thank the medical staff of the University Hospital Leipzig and the staff members of the Coordination Centre for Clinical Trials Leipzig (http://www.kksl.uni-leipzig.de) for helpful discussions. We would also like to thank Luisa Mantovani, head of the department for oncology and hematology at the hospital "St. Georg" (Leipzig), for allowing us to look at patient records. Michael Brosig, Institute for Medical Informatics, Statistics and Epidemiology, University of Leipzig, was very helpful in analyzing the patient records. The work presented in this paper has also benefited from the BMBFfunded Competence Network Malignant Lymphoma (http://www.lymphome.de).

\section{References}

1. Friedman LM, Furberg CD, DeMets DL. Fundamentals of clinical trials. Third Edition. Berlin: Springer; 1998.

2. Pocock SJ. Clinical Trials: A Practical Approach. Chichester: Wiley; 1983.

3. OncoWorkstation. Available at http://www.onco workstation.de.

4. Schmitz N, Glass B, Kloess M, Loeffler M. MegaCHOEP Phase III, DSHNHL 2002-1, 27.02.2003. Available at: http://www.lymphome.de/Gruppen/ DSHNHL/Protokolle/2002-1/index.jsp.

5. Clayton PD. Hospital Information Systems: Clinical Use. In: van Bemmel JH, Musen M, editors. Handbook of Medical Informatics. Heidelberg: Springer; 1997; pp. 331-41.

6. Lodder H, Bakker AR, Zwetsloot JHM. Hospital Information Systems: Technical Choices. In: van Bemmel JH, Musen M, editors. Handbook of Medical Informatics. Heidelberg: Springer; 1997; pp. 343-56.

7. Decruyenaere J, De Turck F, Vanhastel S, Vandermeulen F, Demeester P, de Moor G. On the design of a generic and scalable multilayer software architecture for data flow management in the intensive care unit. Methods Inf Med 2003; 42 (1): 79-88.

8. Degoulet P, Fieschi M. Introduction to Clinical Informatics. Springer; 1999.

9. Geissbühler A, Lovis C, Lamb A, Spahni S. Experience with an XML/HTTP-based federative approach to develop a hospital-side clinical information system. In: Patel V, Rogers R, Haux R, editors. Medinfo 2001, Proceedings of the 10th World Congress on Medical Informatics. Amsterdam: IOS Press; 2001: 735-9.

10. Van De Velde R, Degoulet P. Clinical Information Systems: A Component-Based Approach. Springer; 2003.

11. Lawrence P. Workflow Handbook. New York: John Wiley and Sons; 1997.
12. Leymann F, Roller D. Production workflow: concepts and techniques. Upper Saddle River, N.J.: Prentice Hall PTR; 2000.

13. Dadam P, Reichert M, Kuhn K. Clinical workflows - the killer application for process-oriented information systems? In: Proc. 4th International Conference on Business Information Systems, 2000. Berlin: Springer; 2000; pp. 36-59.

14. Miksch S, Kosara R, Seyfang A. Is Workflow Management Appropriate for Therapy Planning? In: Proc. of EWGLP 2000. Amsterdam: IOS Press; 2001: pp. 53-69.

15. Paton N, editor. Active rules in database systems. Heidelberg: Springer; 1999

16. Hahn EG, Hohenberger W. InTACT: Interdisciplinary Trial for Adjuvant Colon Cancer Treatment (UICC III). Friedrich-Alexander-University; Erlangen-Nuremberg, Germany; 1997.

17. Ritter J, Gadner H, Hermann J, Fink FM. Therapy trial AML-BFM-93. The Society for Pediatric Oncology and Hematology (GPOH); Germany; 1993.

18. Tu SW, Kahn MG, Musen M, Ferguson JC, Shortliffe EH, Fagan LM. Episodic Skeletal-Plan Refinement Based on Temporal Data. Communication of the ACM 1989; 32 (12): 1436-55.

19. Giblin G, Lam R. Programming workflow applications with Domino. R\&D Publications; 2000.

20. IABG. Reference manuals of ProMInanD. Munich: IABG Company; 1999.

21. Remedy Corporation. Action request system 4.0 reference manuals. Remedy Corporation; 2000.

22. Reichert M, Dadam P. ADEPT flex $_{-}$-supporting dynamic changes of workflows without losing control. Journal of Intelligent Information Systems 1998; 10: 93-129.

23. Casati F, Ceri S, Paraboschi S, Pozzi G. Specification and implementation of exceptions in workflow management systems. ACM TODS 1999; 24 : 405-51.

24. Chiu DKW, Li Q, Karlapalem K. Web interfacedriven cooperative exception handling in ADOME workflow management system. Information Systems 2001; 26: 93-120.

25. Liu L, Pu C. Methodical restructuring of complex workflow activities. In: Proc ICDE 1998; IEEE Computer Society Press; 1998: pp. 342-50.

26. Quaglini S, Stefanelli M, Lanzola G, Caporusso V, Panzarasa S. Flexible guideline-based patient careflow systems. Artificial Intelligence in Medicine 2001; 22 (1): 65-80.

27. Wang D, Shortliffe EH. GLEE - a model-driven execution system for computer-based implementation of clinical practice guidelines. Proc AMIA Symp 2002: pp. 855-9.

28. Heller B, Loeffler M, Musen M, Stefanelli M, editors. Computer-Based Support for Clinical Guidelines and Protocols. Proceedings of EWGLP 2000. Amsterdam: IOS Press; 2001.

29. Musen M, Tu SW, Das AK, Shahar Y. EON: A Component-Based Architecture for Automation of Protocol-Directed Therapy. Journal of the American Medical Informatics Association 1996; 3 (6): $367-88$

30. Mueller R, Sergl M, Nauerth U, Dittrich HM, Schoppe D, Pommerening K. TheMPO: A Knowl-
edge-Based System for Therapy Planning in Pediatric Oncology. Computers in Biology and Medicine 1997; 27 (3): 177-200.

31. Mueller R. Event-Oriented Dynamic Adaptation of Workflows: Model, Architecture and Implementation. PhD Thesis; University of Leipzig; 2002.

32. Hripcsak G, Clayton PD, Jenders RA, Cimino JJ, Johnson SB. Design of a clinical event monitor. Comput Biomed Res 1996; 29 (3): 194-221.

33. Loeffler M, Koeppler H, Pfreundschuh M, Havemann K. Integratives Konzept zur Behandlung hochmaligner Non-Hodgkin-Lymphome (Studie A und Studie B). Marburg, Homburg; 1994.

34. Schmitz N. et al. NHL-R1: Multizentrische, offene, nicht randomisierte Therapiestudie zur Bestimmung der maximal praktikablen Dosis bei dreimaliger mittelhoch-dosierter BEAM-Therapie und Blutstammzelltransplantation bei Patienten von 16-60 Jahren mit erstem Rezidiv eines hochmalignen NHL. Kiel; 1996.

35. Birkmann J, Truemper L, Hasenclever D. NHL-R2: Phase I/II-Studienprotokoll für $\mathrm{Pa}-$ tienten mit erstem Therapieversagen und Kontraindikation für ABSCT: CEMP. Nürnberg; 1994.

36. Diehl V et al. Studienprotokolle der Primärtherapie HD4, HD8, HD9 und Studienprotokoll zur Rezidivtherapie HDR1. Köln; 1993

37. Diehl V et al. Studienprotokolle der Primärtherapie HD10, HD11, HD12. Köln, 1998.

38. Pfreundschuh $M$ et al. Studienprotokoll DSHNHL 1999-1A (RICOVER-60). Homburg, 1999.

39. Blobel B. Analysis, Design and Implementation of Secure and Interoperable Distributed Health Information Systems. IOS-Press, Amsterdam; 2002.

40. Saafran C, Perreault LE. Management of Information in Integrated Delivery Networks. In: Shortliffe EH, Perreault LE (eds). Medical Informatics. Computer Applications in Health Care and Biomedicine. Springer, New York, 2001: pp. 359-96.

41. Vlachos M, Stassinopoulos G. Open, Flexible and Portable Secure Web-based Health Applications. In: Blobel B, Pharow P (eds). Advanced Health Telematics and Telemedicine. IOS-Press, Amsterdam, 2003: 41-50.

42. Pfreundschuh M et al. 2-weekly or 3-weekly CHOP Chemotherapy with or without Etoposid for the Treatment of Elderly Patients with Aggressive Lymphomas: Results of the NHL-B2 trial of the DSHNHL. Blood 2004 (DOI 10.1182/blood-2003-06-2095).

Correspondence to:

Ulrike Greiner

Universitüt Leipzig

Institut für Informatik

Augustusplatz 10-11

04109 Leipzig

Germany

E-mail: greiner@informatik.uni-leipzig.de 